\title{
Modified hypothermic circulatory arrest for emergent repair of acute aortic dissection type a: a single-center experience
}

\author{
Hong Qian ${ }^{1 \dagger}$, Jia Hu${ }^{1 \dagger}$, Lei Du², Ying Xue ${ }^{2}$, Wei Meng ${ }^{1}$ and Er-yong Zhang ${ }^{1 *}$
}

\begin{abstract}
Background: Deep hypothermic circulatory arrest (DHCA) with antegrade cerebral perfusion has been historically preferred for organ protection during surgical repair of the acute aortic dissection type A. However, in the past decades, different perfusion-specific strategies with a growing trend to increase the body temperature at circulatory arrest emerged. In this study, we retrospectively analyzed the clinical results of our modified protocol for cardiopulmonary bypass and hypothermia management.

Methods: Between February 2007 and September 2012, 54 consecutive patients suffering from acute aortic dissection type A underwent emergent surgery. All patients received hypothermic circulatory arrest in combination with antegrade cerebral perfusion. The patients were divided into two subsets according to the degree of hypothermia and perfusion strategies: namely the DHCA group and the group of modified hypothermic circulatory arrest (MHCA).

Results: The overall 30-day mortality was $27.8 \%$ and was not significantly different between groups (DHCA, 33.3\%, MHCA, 19\%; $p=0.253$ ). The requirement for blood product transfusion in MHCA patients was significantly less as as compared with the patients in the DHCA group. No difference occurred in the incidence of temporary neurologic dysfunction, dialysis-dependent renal failure, or reexploration for bleeding between two groups of patients. The use of MHCA was identified as a protective factor against the postoperative composite complications $(\mathrm{OR}, 0.78 ; \mathrm{Cl}, 0.52$ to $0.98 ; p=0.04)$ and the prolonged intensive care unit stay $(\mathrm{OR}, 0.8 ; 95 \% \mathrm{Cl}, 0.56$ to $0.98 ; p=0.04)$.

Conclusions: Moderate hypothermia in combination with selective brain perfusion and systemic retrograde perfusion is associated with adequate cerebral and visceral protection, reduced postoperative complications and shortened intensive care unit stay in our series. This modified perfusion strategy may help in improving perioperative outcomes in this particular group of patients.
\end{abstract}

Keywords: Acute aortic dissection, Hypothermia, Circulatory arrest, Aortic surgery

\section{Background}

Acute aortic dissection type A (AADA) is one of the most challenging diseases in cardiovascular surgery. Despite the continuous advances of operative techniques and perfusion-specific technologies during recent years, several international registries still reported an in-hospital mortality rate in patients with AADA of $17 \%-28 \%$ [1-4]. Ischemic visceral organ failure and postoperative neurologic dysfunction

\footnotetext{
*Correspondence: zey16@126.com

${ }^{\dagger}$ Equal contributors

'Department of Cardiovascular Surgery, West China Hospital, Sichuan University, Chengdu, People's Republic of China

Full list of author information is available at the end of the article
}

are closely associated with the significant perioperative mortality and morbidity in surgically treated patients. During past decades, different management of the circulation for the cerebral and visceral organ protection during complicated aortic reconstruction has been developed, however the optimal strategy remains the subject of ongoing debate $[3,5]$.

Deep hypothermic circulatory arrest (DHCA) with selective antegrade cerebral perfusion (ACP) is an effective cerebral protection technique and has widely been applied in the surgical repair of AADA requiring open distal anastomosis. More recently, the absolute 
necessity for deep hypothermia during circulatory arrest once physiologic ACP is provided has been questioned. A series of studies have demonstrated that patients undergoing mild or moderate hypothermic circulatory arrest were presented with a reduction in the duration of cardiopulmonary bypass $(\mathrm{CPB})$, systemic inflammatory responses, organ dysfunction and coagulation disorders [6-10]. However, concerns also grew among the surgeons about the effectiveness of the mild-to-moderate hypothermia for protecting visceral organs and lower body during circulatory arrest, especially when a long arch repair time was required $[2,11,12]$.

We herein present the early results of different perfusion strategies that have been employed at our institution during emergent repair of AADA over a five-year period. Our cerebral and visceral organ protection protocol evolved from the use of DHCA (core temperature $<25^{\circ} \mathrm{C}$ ) with unilateral ACP to modified hypothermic circulatory arrest (MHCA) over the study period. The MHCA protocol consists of moderate hypothermia (core temperature $25^{\circ} \mathrm{C}-28^{\circ} \mathrm{C}$ ) and unilateral ACP combined with episodes of retrograde systemic perfusion during circulatory arrest. This change of paradigm allows us to retrospectively review and assess the safety and outcomes of performing AADA repair with these two perfusion-specific techniques.

\section{Methods}

\section{Patient profile}

Data from two hundred and twenty-two patients with AADA who admitted to our institution between February 2007 and September 2012 were prospectively collected and retrospectively analyzed. Of these patients, 201 patients (90.2\%) were treated surgically, and only 54 patients underwent emergent repair with the aid of hypothermic circulatory arrest, selective ACP. Reasons for turning down from emergent surgery were advanced age, comorbidity and patient refusal. Retrospective data analysis was approved by the Institutional Review Board at West China Hospital in compliance with the Declaration of Helsinki. The need for individual patient consent was waived. The patients were divided into two subsets according to the perfusion protocol during circulatory arrest: namely the DHCA group and the MHCA group. Perioperative characteristics of the patients in both groups are summarized in Table 1.

\section{Operative techniques}

After induction of anaesthesia, the left radial artery \pm one of the femoral arteries were cannulated for continuous measurement of systemic perfusion pressures during cardiopulmonary bypass. Temperature probes were placed for nasopharyngeal and rectal temperature monitoring. Intraoperative transesophageal echocardiography was used in all patients.
Table 1 Preoperative demographics and clinical characteristics

\begin{tabular}{|c|c|c|c|}
\hline Risk factors & $\begin{array}{l}\text { DHCA } \\
(n=33)\end{array}$ & $\begin{array}{l}\text { MHCA } \\
(n=21)\end{array}$ & $p$ Value \\
\hline Age, years & $44.9 \pm 11.7$ & $46.9 \pm 10.7$ & 0.533 \\
\hline Weight, kg & $68.3 \pm 11.6$ & $67.0 \pm 11.3$ & 0.669 \\
\hline Male/Female & $\begin{array}{c}26 / 7 \\
(78.8 \% / 21.2 \%)\end{array}$ & $\begin{array}{c}17 / 4 \\
(81.0 \% / 19.0 \%)\end{array}$ & 1.000 \\
\hline Smoking history & $15(45.5 \%)$ & $10(47.6 \%)$ & 0.876 \\
\hline Hypertension & $14(42.4 \%)$ & $8(38.1 \%)$ & 0.752 \\
\hline Diabetes & $8(24.2 \%)$ & $5(23.8 \%)$ & 0.971 \\
\hline \multicolumn{4}{|l|}{ Etiology } \\
\hline Aneurysmal disease & $28(84.8 \%)$ & 19 (90.5\%) & 0.693 \\
\hline Marfan syndrome & $4(12.1 \%)$ & $1(4.8 \%)$ & 0.638 \\
\hline Others & $1(3.0 \%)$ & $1(4.8 \%)$ & 1.000 \\
\hline Mitral regurgitation & $2(6.1 \%)$ & $0(0 \%)$ & 0.516 \\
\hline Aortic regurgitation & $16(48.5 \%)$ & $8(38.1 \%)$ & 0.638 \\
\hline ASA classification & E3 (E2-E5) & E4 (E3-E5) & 0.606 \\
\hline LVEF & $64.4 \% \pm 5.3 \%$ & $63.4 \% \pm 8.9 \%$ & 0.629 \\
\hline Hemoglobin (g/L) & $124.9 \pm 17.0$ & $123.9 \pm 19.4$ & 0.847 \\
\hline Hematocrit (\%) & $0.38 \pm 0.05$ & $0.37 \pm 0.05$ & 0.733 \\
\hline White blood cell (10 [9]/L) & $9.6 \pm 3.1$ & $8.2 \pm 3.3$ & 0.138 \\
\hline $\mathrm{PaO}_{2} / \mathrm{FiO}_{2}$ ratio & $195.3 \pm 35.1$ & $210.1 \pm 76.5$ & 0.412 \\
\hline Creatinine (umol/L) & $101.6 \pm 48.6$ & $97.6 \pm 31.2$ & 0.736 \\
\hline BUN (mmol/L) & $10.6 \pm 12.8$ & $8.2 \pm 3.9$ & 0.411 \\
\hline Total billirubin (umol/L) & $19.6 \pm 9.6$ & $19.4 \pm 10.6$ & 0.949 \\
\hline $\mathrm{ALT}(\mathrm{IU} / \mathrm{L})$ & $47.4 \pm 84.7$ & $64.1 \pm 119.9$ & 0.537 \\
\hline AST (IU/L) & $48.2 \pm 84.7$ & $40.0 \pm 34.0$ & 0.678 \\
\hline
\end{tabular}

AADA, acute aorta dissection type A; ALT, alanine aminotransferase; ASA, American Society of Anesthesiologists; AST, aspartate aminotransferase; BUN, blood urea nitrogen; DHCA, deep hypothermic circulatory arrest; MHCA, modified hypothermic circulatory arrest; LVEF, left ventricular ejection fraction.

The patient was positioned on the operating table in a supine position. After administration of $300 \mathrm{IU} / \mathrm{Kg}$ heparin, an 8- or 10-mm Gore-Tex graft (Gore \& Associates Inc., Arizona, USA) was anastomosed end-to-side to the right axillary artery with a running $6 / 0$ polypropylene suture. The graft was then cannulated with a $22 \mathrm{~F}$ elongated arterial cannula (Medtronic Corp, Minneapolis, MN) and secured.

All patients were approached through a midline sternotomy, extending the skin incisions a few centimeters above the manubrium. The anterior surface of the head vessels was carefully dissected. The decision for one particular cannulation strategy was made on the basis of preoperative investigations or intraoperative transesophageal echocardiography. When axillary artery alone was not suitable for arterial cannulation or failed to maintain adequate CPB flow, other arterial accesses in combination with or without axillary artery were considered, including 
the femoral artery, the brachiocephalic artery and the left common carotid artery.

The superior vena cava and inferior vena cava (IVC) were cannulated and drained separately for systemic venous return. $\mathrm{CPB}$ was started with a mean flow of $50-60 \mathrm{ml} / \mathrm{kg} / \mathrm{min}$. Systemic cooling was performed using the alpha-stat method for acid-base management. We continued to cool the patient and kept the mixed venous oxygen saturation at $75 \%-85 \%$.

After reaching the target temperature, the patient was placed in Trendelenburg position, and the head was cooled topically. Selective unilateral ACP was initiated by clamping the brachiocephalic trunk before circulatory arrest and opening of the aorta. The extent of resection was determined by the extent of the aortic pathology and the location of entry site or reentry tear. If the aneurysm was confined to ascending aorta, only this segment was resected and a simple open distal anastomosis was performed. If the aortic arch had aneurysmal involvement, partial or total arch replacement was performed with or without an elephant trunk procedure. Upon completion of the circulatory arrest, the grafts were de-aired prior to restitution of full body perfusion and rewarming. Proximal aortic reconstruction as well as any concomitant procedures was conducted during the rewarming period.

\section{Temperature control and cardiopulmonary bypass management}

In the DHCA group, patients received unilateral ACP with a constant flow rate of $8-10 \mathrm{ml} / \mathrm{kg} / \mathrm{min}$ to maintain a perfusion pressure of $35-70 \mathrm{mmHg}$. During circulatory arrest, the nasopharyngeal temperature was kept at $18-20^{\circ} \mathrm{C}$ and rectal temperature was kept at $24^{\circ} \mathrm{C}-25^{\circ} \mathrm{C}$. Patients in the MHCA group were managed with moderate hypothermia (nasopharyngeal temperature $24^{\circ} \mathrm{C}-25^{\circ} \mathrm{C}$ and rectal temperature $\left.27^{\circ} \mathrm{C}-28^{\circ} \mathrm{C}\right)$, selective ACP $(7-12$ $\mathrm{ml} / \mathrm{kg} / \mathrm{min}$ with perfusion pressure maintained at 45-55 $\mathrm{mmHg}$ ) and episodes of systemic retrograde perfusion during circulatory arrest (2-3 minutes per time with a flow of $5-8 \mathrm{ml} / \mathrm{kg} / \mathrm{min}$ in every $15-20$ minutes). The systemic retrograde perfusion was carried out through the IVC cannulation. After snaring the IVC by surgeons, perfusionist partially clamped the IVC return line to maintain the central venous pressure as high as $10-12 \mathrm{mmHg}$. As the arterial pressure of the lower body was less than $5 \mathrm{mmHg}$ during the circulatory arrest, the elevated central venous pressure was adequate to drive a short period of the systemic retrograde perfusion. A $15 \%-20 \%$ drop of saturation of venous oxygen was regarded as an efficient retrograde perfusion.

\section{Definitions}

Definitions were according to the Society of Thoracic Surgery National Database specifications (http://www.sts. org/sites/default/files/documents/word/STSAdultCVData
SpecificationsV2_73\%20with\%20correction.pdf). Emergent surgery was regarded as operation performed within 24 hours of hospital admission for cardiovascular instability. Acute aortic dissection is defined as occurring within 2 weeks of onset of initial symptoms [13]. Neurologic outcome was categorized as permanent neurologic dysfunction (PND) and temporary neurologic dysfunction (TND) $[8,14]$. PND was defined as the presence of a new postoperative focal (stroke) or global (Parkinsonism, coma, gait disturbance) neurologic deficit and persisting at discharge. TND was regarded as the occurrence of postoperative agitation, confusion, delirium, obtundation or a transient focal neurologic deficit (resolution within 72 hours) without any evidence of new structural abnormality on computed tomography or magnetic resonance imaging.

\section{Statistical analysis}

Continuous variables in the tables and texts are expressed as mean \pm standard deviation and categorical data as proportions. Categorical variables were compared using Chi-square test or Fisher's exact test. Independent continuous variables were compared by unpaired Student's $t$ test for normally distributed data between two groups, and Mann-Whitney U-test was used for the comparison of parameters that did not exhibit a normal distribution. Variables with a univariate $p<0.2$ or those of known clinical importance for 30-day mortality and postoperative outcome events were included in a stepwise multivariate logistic regression model to calculate odds ratios (OR) and its 95\% confidence intervals (CI) (Table 2). As others did before [15], we combined re-exploration for bleeding, dialysis-dependent renal failure, postoperative pneumonia, mediastinal infection, sepsis, tracheotomy and TND as the composite postoperative complications to increase the sensitivity for identifying risk factors. All $p$ values less than 0.05 were considered statistically significant. All statistical

\section{Table 2 Preoperative factors used in logistic regression} analyses

\begin{tabular}{|c|c|}
\hline Preoperative factors & Intraoperative factors \\
\hline Age & Duration of cardiopulmonary bypass \\
\hline Gender & Cross-clamp time \\
\hline Hypertension & Circulatory arrest time $\geq 40$ minutes \\
\hline Diabetes & $\begin{array}{l}\text { Core temperature during circulatory } \\
\text { arrest }\end{array}$ \\
\hline Left ventricular ejection fraction & Deep hypothermic circulatory arrest \\
\hline ASA classification & $\begin{array}{l}\text { Modified hypothermic circulatory } \\
\text { arrest }\end{array}$ \\
\hline \multicolumn{2}{|l|}{ Preoperative $\mathrm{PaO}_{2} / \mathrm{FiO}_{2}$ Ratio } \\
\hline \multicolumn{2}{|l|}{ Renal insufficiency } \\
\hline Liver function impairment & \\
\hline
\end{tabular}

ASA, American Society of Anesthesiologist. 
analyses were performed using Statistical Package for Social Sciences (SPSS) version 16.0 (SPSS Inc, Chicago, IL, USA).

\section{Results}

A total of 54 patients underwent emergency surgery for AADA. The underlying chronic pathology was aneurysmal disease (88.9\%), Marfan syndrome (9.3\%) and other etiology $(1.8 \%)$, such as infection or porcelain aorta. The mean age of patients in the series was $45.7 \pm 11.2$ years (range, 22 to 75 years), 43 were male and 11 were female. Twenty-two $(40.7 \%)$ patients and thirteen patients (24.1\%) had a history of hypertension and diabetes, respectively. Hypothermic circulatory arrest and unilateral ACP were used in all patients and 21 patients in MHCA group underwent additional episodes of systemic retrograde perfusion during circulatory arrest.

\section{Cardiopulmonary bypass data and operative details}

The mean $\mathrm{CPB}$ time in all patients was $256.0 \pm 84.62$ minutes (range 131 to 566 minutes) and the mean aortic cross-clamp time was $149.5 \pm 41.6$ minutes (range 62 to 294 minutes). The mean circulatory arrest time was significantly longer for patients in DHCA group than for patients undergoing MHCA (44.5 minutes versus 32.1 minutes, $\mathrm{p}=0.005$; Table 3 ). Forty patients (74.1\%) underwent total arch replacement, including 35 patients with a stage I elephant trunk procedure. Thirty patients (55.6\%) underwent ascending aorta replacement, and one patient (3\%) underwent hemiarch replacement. Proximal aortic procedures included Bentall procedure in 15 patients (27.8\%), Cabrol procedure in 6 patients (11.1\%), and Wheat procedure (1.9\%). As demonstrated in Table 3, the different arch interventions equally distributed between the two groups. An additional 2 patients (3.7\%) underwent aortic valve reconstruction and 2 patients (3.7\%) received concomitant mitral valve interventions.

\section{Perioperative complications and outcome events}

The overall incidence of TND was $9.2 \%$ and no patients had permanent neurologic deficits. The number of patients developing complicating pneumonia were significantly greater in the DHCA group than those in the MHCA group (57.6\% versus $23.8 \%, p=0.015)$. Although the incidence of reexploration for bleeding was not significantly different between groups, the need for blood product transfusion in MHCA patients was significantly less as compared to the patients in the DHCA group (Table 4). Dialysis was required postoperatively in 6 patients (11.1\%). Unadjusted differences between the two groups were not statistically different. Multivariate analysis identified age (OR, 2.74; 95\% CI, 2.12 to 3.76; $p=0.004$ ) and CPB times (OR, 1.98; CI, 1.82 to $2.44 ; p=0.015$ ) as independent predictors for composite complications, and the use of MHCA was determined as a protective factor against the composite postoperative complications (OR, 0.78; $\mathrm{CI}, 0.52$ to $0.98 ; p=0.04$ ).

The lactate level early after surgery in both groups was not significantly different (DHCA group: $9.27 \pm 4.93 \mathrm{mmol} / \mathrm{L}$ versus MHCA group: $9.98 \pm 4.75 \mathrm{mmol} / \mathrm{L} ; p=0.41)$. Similarly, no differences in the parameters of renal and liver function, including creatinine, blood urea nitrogen, total bilirubin, and aminotransferases, in DHCA and MHCA patients were observed (Table 4). However, in patients who underwent circulatory arrest for more than 40 minutes, the renal and liver function of MHCA patients $(n=9)$ was significantly improved as compared to the parameters in DHCA patients $(\mathrm{n}=15)$ (Table 5).

Table 3 Cardiopulmonary bypass data and surgical procedures

\begin{tabular}{|c|c|c|c|c|}
\hline Intraoperative data & $\begin{array}{c}\text { All } \\
(n=54)\end{array}$ & $\begin{array}{l}\text { DHCA } \\
(n=33)\end{array}$ & $\begin{array}{l}\text { MHCA } \\
(n=21)\end{array}$ & ${ }^{*} p$ Value \\
\hline \multicolumn{5}{|l|}{ Temperature during HCA } \\
\hline Nasopharynx $\left({ }^{\circ} \mathrm{C}\right)$ & $20.3 \pm 3.9$ & $17.6 \pm 2.4$ & $24.6 \pm 0.4$ & $<0.001$ \\
\hline Rectal $\left({ }^{\circ} \mathrm{C}\right)$ & $22.5 \pm 3.8$ & $19.7 \pm 2.3$ & $26.4 \pm 1.1$ & $<0.001$ \\
\hline Operative time(minutes) & $511.7 \pm 108.3$ & $534.2 \pm 95.9$ & $476.5 \pm 119.3$ & 0.056 \\
\hline Duration of CPB (minutes) & $256.0 \pm 84.3$ & $289.5 \pm 79.9$ & $203.3 \pm 61.9$ & $<0.001$ \\
\hline Cross-clamp (minutes) & $149.5 \pm 41.6$ & $161.9 \pm 34.9$ & $130.2 \pm 44.6$ & 0.005 \\
\hline Circulatory arrest (minutes) & $39.6 \pm 16.6$ & $44.5 \pm 18.4$ & $32.1 \pm 9.7$ & 0.006 \\
\hline \multicolumn{5}{|l|}{ Surgical Procedures } \\
\hline Total aortic arch replacement & $40(74.1 \%)$ & $26(78.8 \%)$ & $14(66.7 \%)$ & 0.434 \\
\hline Elephant trunk & $35(66.7 \%)$ & $22(66.7 \%)$ & $13(61.9 \%)$ & 0.524 \\
\hline Ascending aorta replacement & $30(55.6 \%)$ & $17(51.5 \%)$ & 13(61.9\%) & 0.433 \\
\hline Bentall procedure & $15(27.8 \%)$ & $8(24.2 \%)$ & $7(33.3 \%)$ & 0.399 \\
\hline Cabrol procedure & $6(11.1 \%)$ & $5(15.2 \%)$ & $1(4.8 \%)$ & 0.276 \\
\hline
\end{tabular}

CPB, cardiopulmonary bypass; DHCA, deep hypothermic circulatory arrest; MHCA, modifies hypothermic circulatory arrest. *DHCA versus MHCA. 
Table 4 Postoperative morbidity and mortality

\begin{tabular}{|c|c|c|c|}
\hline $\begin{array}{c}\text { Clinical characteristics } \\
\text { \& outcomes }\end{array}$ & $\begin{array}{l}\text { DHCA } \\
(n=33)\end{array}$ & $\begin{array}{l}\text { MHCA } \\
(n=21)\end{array}$ & $p$ Value \\
\hline In-hospital mortality & $9(27.3 \%)$ & $3(14.3 \%)$ & 0.329 \\
\hline 30-day mortality & $11(33.3 \%)$ & $4(19.0 \%)$ & 0.253 \\
\hline Re-exploration for bleeding & $2(6.1 \%)$ & $4(19.0 \%)$ & 0.193 \\
\hline Renal failure-dialysis & $5(15.2 \%)$ & $1(4.8 \%)$ & 0.386 \\
\hline Pneumonia & $19(57.6 \%)$ & $5(23.8 \%)$ & 0.015 \\
\hline Tracheotomy & 13(39.4\%) & $2(9.5 \%)$ & 0.017 \\
\hline Mediastinal infection & $3(9.1 \%)$ & $1(4.8 \%)$ & 1.000 \\
\hline Sepsis & $3(9.1 \%)$ & $0(0 \%)$ & 0.282 \\
\hline TND & $4(12.1 \%)$ & $1(4.8 \%)$ & 0.638 \\
\hline PND & $0(0 \%)$ & $0(0 \%)$ & 1.000 \\
\hline Length of ICU stay, days & $7.4 \pm 4.5$ & $4.5 \pm 1.9$ & 0.010 \\
\hline Length of hospital stay, days & $18.6 \pm 6.2$ & $17.4 \pm 5.5$ & 0.556 \\
\hline Mechanical ventilation, hours & $141.2 \pm 125.4$ & $75.1 \pm 68.0$ & 0.035 \\
\hline Red blood cell transfusion, IU & $14.6 \pm 8.2$ & $10.3 \pm 7.3$ & 0.045 \\
\hline FFP transfusion, ml & $2590.9 \pm 1479.0$ & $1419.1 \pm 1061.5$ & 0.003 \\
\hline Platelet transfusion, IU & $1.6 \pm 0.9$ & $0.8 \pm 1.1$ & 0.007 \\
\hline $\begin{array}{l}\text { Drainage during first72h, ml } \\
\qquad \mathrm{PaO}_{2} / \mathrm{FiO}_{2} \text { Ratio }\end{array}$ & $2040.2 \pm 1075.0$ & $1543.8 \pm 1071.0$ & 0.116 \\
\hline Postoperative day 1 & $170.0 \pm 43.9$ & $163.5 \pm 40.7$ & 0.718 \\
\hline Postoperative day 3 & $177.1 \pm 56.3$ & $228.8 \pm 79.9$ & 0.017 \\
\hline \multicolumn{4}{|l|}{ Lactate $(\mathrm{mmol} / \mathrm{L})$} \\
\hline Postoperative 1 hours & $9.3 \pm 4.9$ & $10.00 \pm 4.8$ & 0.410 \\
\hline Postoperative 4 hours & $5.9 \pm 3.8$ & $5.9 \pm 3.4$ & 0.983 \\
\hline Creatinine (umol/L) day 3 & $249.2 \pm 237.7$ & $203.5 \pm 178.7$ & 0.480 \\
\hline BUN (umol/L) day 3 & $25.5 \pm 14.6$ & $23.0 \pm 13.1$ & 0.538 \\
\hline Total bilirubin (umol/L) day 3 & $53.9 \pm 59.0$ & $35.3 \pm 20.2$ & 0.226 \\
\hline ALT (IU/L) day 3 & $103.2 \pm 91.4$ & $102.0 \pm 108.0$ & 0.967 \\
\hline AST (IU/L) day 3 & $97.6 \pm 107.7$ & $78.6 \pm 70.1$ & 0.502 \\
\hline
\end{tabular}

TND, temporary neurologic dysfunction; PND, permanent neurologic dysfunction; ICU, intensive care unit; BUN, blood urea nitrogen; FFP, fresh frozen plasma; ALT, alanine aminotransferase; AST, aspartate aminotransferase.

\section{Risk factors for prolonged hospitalization and early mortality}

The average hospital stay was $18.1 \pm 5.9$ days, and no significant difference between the DHCA group and the MHCA group was observed (18.6 \pm 6.2 days versus 17.4 \pm 5.5 days, $p=0.556$ ). However, the average intensive care unit (ICU) stay was significantly shorter for patients in the MHCA group than patients from the DHCA group ( $4.5 \pm 1.9$ days versus $7.4 \pm 4.5$ days, $p=0.010)$. Compared with patients who underwent deep hypothermia, patients in the MHCA group are less likely to have tracheotomy (9.5\% versus $39.4 \%, p=0.017)$ and require shorter time of mechanical assisted ventilation $(75.1 \pm 68.0$ hours versus 141.2 \pm 125.4 hours, $p=0.035$ ). The use of MHCA was
Table $\mathbf{5}$ Visceral function in patients with prolonged circulatory arrest

\begin{tabular}{lccc}
\hline Parameters & $\begin{array}{c}\text { DHCA } \\
(\mathbf{n = 1 5})\end{array}$ & $\begin{array}{c}\text { MHCA } \\
(\mathbf{n = 9})\end{array}$ & $\boldsymbol{p}$ Value \\
\hline Lactate (mmol/L) & & & \\
Postoperative 1 hours & $9.5 \pm 3.7$ & $10.0 \pm 3.0$ & 0.09 \\
Postoperative 4 hours & $6.1 \pm 2.9$ & $5.8 \pm 3.4$ & 0.32 \\
Creatinine (umol/L) day 3 & $328.8 \pm 281.6$ & $233.1 \pm 186.1$ & $\mathbf{0 . 0 0 7}$ \\
BUN (umol/L) day 3 & $27.1 \pm 10.6$ & $23.9 \pm 9.1$ & $\mathbf{0 . 0 4 4}$ \\
Total bilirubin (umol/L) day 3 & $55.0 \pm 26.3$ & $36.6 \pm 23.4$ & $\mathbf{0 . 0 1 0}$ \\
ALT (IU/L) day 3 & $119.2 \pm 34.7$ & $96.5 \pm 49.8$ & $\mathbf{0 . 0 3 9}$ \\
AST (IU/L) day 3 & $97.8 \pm 82.6$ & $81.3 \pm 69.0$ & $\mathbf{0 . 0 1 8}$ \\
\hline
\end{tabular}

BUN, blood urea nitrogen; ALT, alanine aminotransferase; AST, aspartate aminotransferase.

determined to be the only protective factor against the prolonged ICU stay by multivariate regression analysis (OR, 0.8; 95\% CI, 0.56 to $0.98 ; p=0.04$ ).

In-hospital mortality observed in our study was $22.2 \%$ $(n=12)$, comparable to the published data from other similar case series $[1,4,16,17]$. Thirty-day mortality for the entire cohort was $27.8 \%(n=15)$ and no significant difference between groups was observed $(\mathrm{p}=0.263)$. Multivariate analysis identified preoperative American Society of Anesthesiologists classification (OR, 3.1; 95\% $\mathrm{CI}, 1.062$ to $9.258 ; p=0.019$ ), duration of $\mathrm{CPB}$ (OR, $1.011 ; 95 \% \mathrm{CI}, 1.002$ to $1.019 ; p<0.05)$ as independent predictors of postoperative 30-day mortality.

\section{Discussion}

For past two decades, DHCA in combination with either continuous antegrade or retrograde cerebral perfusion has gained its popularity due to the improved postoperative outcomes as compared with DHCA alone. As the real benefits of retrograde cerebral perfusion are not formally accepted, the combination of deep hypothermia and ACP during circulatory arrest was applied in our hospital (since 2007) and many other high-volume aortic centers. In recent years, the "warmer" temperature at circulatory arrest has been preferred as the safety of mild-to-moderate hypothermic circulatory arrest and the beneficial effects of avoiding deep hypothermia have been reported by a series of studies [6-10]. Since 2010, circulatory arrest in our institution has been obtained at a core temperature of $26^{\circ} \mathrm{C}-28^{\circ} \mathrm{C}$. However, the avoidance of deep core temperature may be at the expense of an increased risk of the ischemic injuries of visceral organs and spinal cord. Thus, we modified our perfusion protocol by adding a short duration of retrograde systemic perfusion during circulatory arrest.

In the current study, we focused on assessing the effectiveness of our modified perfusion protocol (moderate hypothermia $26.4 \pm 1.1^{\circ} \mathrm{C}, \mathrm{ACP}$ and retrograde systemic 

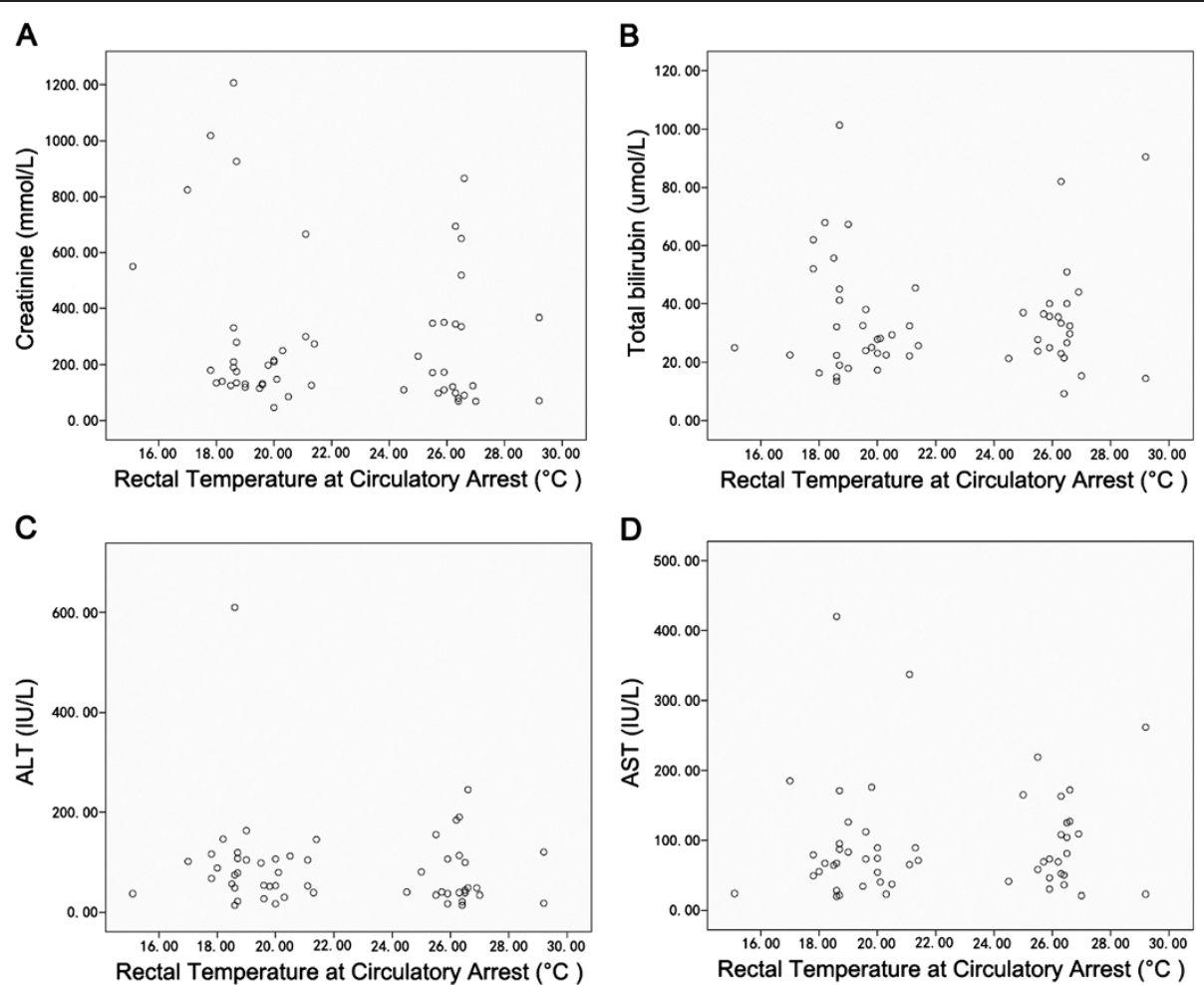

Figure 1 The relationship between visceral organ function and core temperature at circulatory arrest. Scatter plots showing no significant relationship between the early postoperative level of creatinine (A), total bilirubin (B), alanine aminotransferase (ALT, C), and aspartate aminotransferase (AST, D), and rectal temperature at circulatory arrest.

perfusion) for cerebral and visceral protection in the emergent repair of AADA, by comparing outcomes with a cohort that underwent similar surgical interventions at deep hypothermia $\left(19.7 \pm 2.3^{\circ} \mathrm{C}\right)$. Analysis of the entire study population demonstrated that patients undergoing AADA surgery at moderate levels of hypothermia had a trend of reduced in-hospital and 30-day mortality compared with the patients in the DHCA group, but the difference was not statistically significant. The duration of $\mathrm{CPB}$ and preoperative American Society of Anesthesiologists classification were identified as independent predictors for early mortality in this study, similar to data published by Czerny and colleagues [18]. Several risk factors for postoperative mortality that have been identified by other studies, including age, preoperative respiratory failure (oxygenation index $<200$ ), renal insufficiency, level of hypothermia, left ventricular ejection fraction $[2,4-12,19]$, were not found to be the significant predictors for 30-day mortality in our series.

The occurrence of neurological dysfunction after aortic surgery has been shown to be associated with longer ventilator requirements, prolonged ICU stay and higher in-hospital mortality [3,5]. According to the published literature, the overall incidence of TND in AADA patients undergoing hypothermic circulatory arrest and selective cerebral perfusion varies between $2.5-16 \%$ and PND with a reported incidence of $4-24 \%[2,12,15-17,19]$. The risk of postoperative neurologic dysfunction was reported to be significantly influenced by the site of arterial cannulation and could be greatly reduced by cannulating a graft sewn to the right axillary artery $[5,7,15]$. All patients in this study were cannulated through an 8-10 mm Gore-Tex graft sewn to the axillary artery and then received ACP. Therefore, a low postoperative TND rate (9.5\%) with no case of PND observed in our series was not unexpected. Previous studies have identified predictors of the negative neurologic outcomes in surgically treated AADA patients as age, history of cerebrovascular adverse events, renal insufficiency, prolonged hypothermic circulatory arrest time ( $>40$ minutes), and $\mathrm{CPB}$ time $[2,4,12,15,18,19]$. However, in the current study, these risk factors were not found to be independent predictors for postoperative neurologic dysfunction. The reason for this might be attributed to the small sample size of our series, and probably, the surgical procedures in almost all cases were completed within the safety time limits during circulatory arrest.

The reduction of ventilator requirements in the MHCA patients translated into a significant shortened ICU length of stay compared with patients in the DHCA group. The reduction of ventilation requirements may be largely due to the significant shorter $\mathrm{CPB}$ times observed in the MHCA group (203.3 \pm 61.9 minutes) 
as compared to the DHCA group (289.5 \pm 79.9 minutes; $p<0.001)$. It is well known that the extensive cooling and rewarming periods in deep hypothermia not only contributed to the longer duration of $\mathrm{CPB}$ and subsequent deep hypothermia-related vasoconstriction, coagulopathy, but also leads to a secondary vasodilation with increased interstitial edema and reperfusion injury during the extended rewarming phase [11]. Therefore, as we speculated, longer CPB time-induced pulmonary edema and prolonged mechanical ventilation may contribute to the higher incidence of postoperative pneumonia in patients underwent deep hypothermia (DHCA group: 57.6\% versus MHCA group: $23.8 \% ; p=0.015)$. Although the rate of reexploration for bleeding between the two groups was not significantly different, the requirements for blood product transfusion in DHCA patients were markedly higher compared with the MHCA group. This result suggests that the use of deep hypothermia was more likely to be associated with the disturbance of patients' coagulation system.

Theoretically, a cooler temperature could be related with better visceral organ protection, especially when a prolonged duration of lower body circulatory arrest is required. However, from our results, the postoperative renal and liver function in patients underwent moderate hypothermia were similar to those in the DHCA patients. As demonstrated in the scatter plots (Figure 1), no significant relationship between the rectal temperatures at circulatory arrest and the level of creatinine, total bilirubin and aminotransferases were observed. We suggest that in the DHCA group, the longer duration of $\mathrm{CPB}$ and the higher requirements for blood products transfusion may outweigh the beneficial effects provided by the lower temperature. Moreover, all patients in the MHCA group have received episodes of systemic retrograde perfusion during circulatory arrest. It is well known that human venous valves are largely distributed in veins in muscle-skeletal system rather than in visceral organs. During retrograde perfusion, the venous blood would firstly redistribute to veins without valves, such as venous system in liver, kidney and spinal cord, and therefore a better protection of these visceral organs in lower body might be achieved. Although the potential benefit of this method for lower body protection remains to be confirmed by large randomized trials, we may speculate its beneficial effects from the results of our series that the postoperative lactate level in MHCA patients was not inferior to that in DHCA patients, and visceral organ function in MHCA patients who underwent hypothermic circulatory arrest for longer than 40 minutes was significantly improved as compared with the parameters in the DHCA group.

The main limitations of the current study are its retrospective nature, and the absence of a control group of patients who underwent moderate hypothermic circulatory arrest without systemic retrograde perfusion. We acknowledge that without a direct comparison, the real benefits of our modified perfusion strategy for lower body protection remains to be confirmed. However, our results have indicated that AADA patients could benefit from "warmer" hypothermia during circulatory arrest. Furthermore, we cannot exclude the possibility that the improved surgical expertise might have an effect on outcomes, and a propensity score analysis with an enlarged sample size might be performed to limit any influence of the selection bias on our results.

\section{Conclusions}

In summary, data from the current study support the safety and effectiveness of our modified perfusion strategy for cerebral and visceral organ protection during emergent repair of AADA. When directly compared with patients undergoing aortic reconstruction at deep hypothermia, no difference in early postoperative mortality, adverse neurologic outcomes and visceral organ dysfunction was observed. The reduction in blood product transfusion, respiratory complications and ICU stay suggest AADA patients might benefit more from the moderate hypothermia during circulatory arrest. Future studies will investigate the potential benefits of the systemic retrograde perfusion for lower body protection during circulatory arrest.

\section{Abbreviations}

AADA: Acute aortic dissection type A; ACP: Antegrade cerebral perfusion; CPB: Cardiopulmonary bypass; DHCA: Deep hypothermic circulatory arrest; ICU: Intensive care unit; IVC: Inferior vena cava; MHCA: Modified hypothermic circulatory arrest; PND: Permanent neurologic dysfunction; TND: Temporary neurologic dysfunction.

\section{Competing interests}

The authors declare that they have no competing interests.

\section{Authors' contributions}

All manuscript was written by $\mathrm{HQ}$ and $\mathrm{J}$, and all the data were collected by the following surgeons and perfusionists, HQ, JH, LD, YX, WM and EYZ. The collected data were promised by all authors. All authors read and approved the final manuscript.

\section{Author details}

'Department of Cardiovascular Surgery, West China Hospital, Sichuan University, Chengdu, People's Republic of China. ${ }^{2}$ Department of Anesthesiology, West China Hospital, Sichuan University, Chengdu, Sichuan, People's Republic of China.

Received: 16 December 2012 Accepted: 30 April 2013 Published: 9 May 2013

\section{References}

1. Tsai TT, Trimarchi S, Nienaber CA: Acute aortic dissection: perspectives from the International Registry of Acute Aortic Dissection (IRAD). Eur J Vasc Endovasc Surg 2009, 37:149-159.

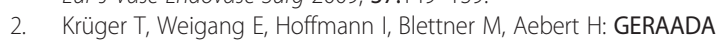
Investigators. Cerebral protection during surgery for acute aortic dissection type A: results of the German Registry for Acute Aortic Dissection Type A (GERAADA). Circulation 2011, 124:434-443.

3. Krüger T, Conzelmann LO, Bonser RS, Borger MA, Czerny M, Wildhirt S, Carrel T, Mohr FW, Schlensak C, Weigang E: Acute aortic dissection type A. Br J Surg 2012, 99:1331-1344

4. Pacini D, Leone A, Belotti LM, Fortuna D, Gabbieri D, Zussa C, Contini A, Di Bartolomeo R, on behalf of RERIC (Emilia Romagna Cardiac Surgery Registry) 
Investigators: Acute type A aortic dissection: significance of multiorgan malperfusion. Eur J Cardiothorac Surg 2012. Epub ahead of print.

5. Bonser RS, Ranasinghe AM, Loubani M, Evans JD, Thalji NM, Bachet JE, Carrel TP, Czerny M, Di Bartolomeo R, Grabenwöger M, Lonn L, Mestres CA, Schepens MA, Weigang E: Evidence, lack of evidence, controversy, and debate in the provision and performance of the surgery of acute type $A$ aortic dissection. J Am Coll Cardiol 2011, 58:2455-2474.

6. Urbanski PP, Lenos A, Bougioukakis P, Neophytou I, Zacher M, Diegeler A: Mild-to-moderate hypothermia in aortic arch surgery using circulatory arrest: a change of paradigm? Eur J Cardiothorac Surg 2012, 41:185-191.

7. Leshnower BG, Myung RJ, Thourani VH, Halkos ME, Kilgo PD, Puskas JD, Chen EP: Hemiarch replacement at $28^{\circ} \mathrm{C}$ : an analysis of mild and moderate hypothermia in 500 patients. Ann Thorac Surg 2012, 93:1910-1915.

8. Misfeld M, Leontyev S, Borger MA, Gindensperger O, Lehmann S, Legare JF, Mohr FW: What is the best strategy for brain protection in patients undergoing aortic arch surgery? A single center experience of 636 patients. Ann Thorac Surg 2012, 93:1502-1508.

9. Zierer A, El-Sayed Ahmad A, Papadopoulos N, Moritz A, Diegeler A, Urbanski PP: Selective antegrade cerebral perfusion and mild $\left(28^{\circ} \mathrm{C}-30^{\circ} \mathrm{C}\right)$ systemic hypothermic circulatory arrest for aortic arch replacement: Results from 1002 patients. J Thorac Cardiovasc Surg 2012, 144:1042-1450.

10. Numata S, Tsutsumi Y, Monta O, Yamazaki S, Seo H, Sugita R, Yoshida S, Ohashi H: Aortic arch repair with antegrade selective cerebral perfusion using mild to moderate hypothermia of more than $28^{\circ} \mathrm{C}$. Ann Thorac Surg 2012, 94:90-95.

11. Kamiya H, Hagl C, Kropivnitskaya I, Böthig D, Kallenbach K, Khaladj N, Martens A, Haverich A, Karck M: The safety of moderate hypothermic lower body circulatory arrest with selective cerebral perfusion: a propensity score analysis. J Thorac Cardiovasc Surg 2007, 133:501-509.

12. Bakhtiary F, Dogan S, Zierer A, Dzemali O, Oezaslan F, Therapidis P, Detho F, Wittlinger T, Martens S, Kleine P, Moritz A, Aybek T: Antegrade cerebral perfusion for acute type A aortic dissection in 120 consecutive patients. Ann Thorac Surg 2008, 85:465-469.

13. Hiratzka LF, Bakris GL, Beckman JA, Bersin RM, Carr VF, Casey DE Jr, Eagle KA, Hermann LK, Isselbacher EM, Kazerooni EA, Kouchoukos NT, Lytle BW, Milewicz DM, Reich DL, Sen S, Shinn JA, Svensson LG, Williams DM: ACCF/ AHA/AATS/ACR/ASA/SCA/SCAI/SIR/STS/SVM guidelines for the diagnosis and management of patients with thoracic aortic disease. J Am Coll Cardiol 2010, 55:e27-e129.

14. Ergin MA, Galla JD, Lansman L, Quintana C, Bodian C, Griepp RB: Hypothermic circulatory arrest in operations on the thoracic aorta. Determinants of operative mortality and neurologic outcome. J Thorac Cardiovasc Surg 1994, 107:788-797.

15. Haldenwang PL, Wahlers T, Himmels A, Wippermann J, Zeriouh M, Kröner A, Kuhr $\mathrm{K}$, Strauch J: Evaluation of risk factors for transient neurological dysfunction and adverse outcome after repair of acute type A aortic dissection in 122 consecutive patients. Eur J Cardiothorac Surg 2012, 42:e115-120.

16. Legras A, Bruzzi M, Nakashima K, Hillion ML, Loisance D, Kirsch M: Colder Is better during hypothermic circulatory arrest for acute type A aortic dissection. Scand Cardiovasc J 2012. Epub ahead of print.

17. Stamou SC, Hagberg RC, Khabbaz KR, Stiegel MR, Reames MK, Skipper E, Nussbaum M, Lobdell KW: Is advanced age a contraindication for emergent repair of acute type A aortic dissection? Interact Cardiovasc Thorac Surg 2010, 10:539-544.

18. Czerny M, Fleck T, Zimpfer D, Dworschak M, Hofmann W, Hutschala D, Dunkler D, Ehrlich M, Wolner E, Grabenwoger M: Risk factors of mortality and permanent neurologic injury in patients undergoing ascending aortic and arch repair. J Thorac Cardiovasc Surg 2003, 126:1296-1301.

19. Trimarchi S, Eagle KA, Nienaber CA, Rampoldi V, Jonker FH, De Vincentiis C, Frigiola A, Menicanti L, Tsai T, Froehlich J, Evangelista A, Montgomery D, Bossone E, Cooper JV, Li J, Deeb MG, Meinhardt G, Sundt TM, Isselbacher EM, International Registry of Acute Aortic Dissection Investigators: Role of age in acute type A aortic dissection outcome: report from the International Registry of Acute Aortic Dissection (IRAD). J Thorac Cardiovasc Surg 2010, 140:784-789.

doi:10.1186/1749-8090-8-125

Cite this article as: Qian et al: Modified hypothermic circulatory arrest for emergent repair of acute aortic dissection type a: a single-center experience. Journal of Cardiothoracic Surgery 2013 8:125.

\section{Submit your next manuscript to BioMed Central and take full advantage of:}

- Convenient online submission

- Thorough peer review

- No space constraints or color figure charges

- Immediate publication on acceptance

- Inclusion in PubMed, CAS, Scopus and Google Scholar

- Research which is freely available for redistribution

Submit your manuscript at www.biomedcentral.com/submit
() Biomed Central 